\title{
DESENHO DE POLIGONOS E SEQUENCIAMENTO DE BLOCOS DE MINÉRIO PARA PLANEJAMENTO DE CURTO PRAZO EM FUNÇÃO DA DISTRIBUIÇÃO DOS TEORES PARA MINERAÇÃO A CÉU ABERTO*
}

\section{Resumo}

Augusto Andrés Torres Toledo ${ }^{1}$ João Felipe Coimbra Leite Costa ${ }^{2}$ Diego Machado Marques ${ }^{3}$

$\mathrm{Na}$ mineração a céu aberto, na etapa de planejamento a curto prazo são desenhados constantemente polígonos usados no planejamento de produção, onde geralmente 0 processo de desenho é manual, demandando o tempo e esforço dos planejadores. Os polígonos gerados no planejamento de curto prazo devem apresentar a menor variabilidade possível entre eles, para que as restrições da planta de processamento sejam atendidas. Desenhar polígonos de curto prazo de forma automática, sequenciar todos os blocos de minério em cada polígono, e conectar um polígono a outro é o foco deste trabalho. Para isso, foi feito uso de algoritmos genéticos e dinâmicos, desenvolvidos em linguagem de programação Python e operacionalizados no software geoestatístico SGeMS. Gerou-se múltiplas iterações para cada um dos avanços individuais de blocos de minério, gerando regiões ou polígonos, e selecionando as regiões de menor variabilidade de teores. As funções de distribuição de probabilade dos teores de cada avanço foram comparadas a função da distribuição de teores global do corpo de minério, os resultados mostram que os polígonos possuem distribuições semelhantes à distribuição de referência, então foi possível sequenciar os blocos de forma operacional garantindo quasi-estacionaridade dos parâmetros.

Palavras-chave: Planejamento de Curto prazo; Sequenciamento de blocos; Estocástico; Função de distribuição provável; Algoritmo genético.

\section{DESIGN OF POLYGONES AND SEQUENCING OF ORE BLOCKS FOR SHORT TERM PLANNING IN FUNCTION OF THE DISTRIBUTION OF THE GRADE OF OPEN PIT MINING}

\begin{abstract}
In open-pit mining, in the short-term planning stage, production polygons are constantly drawn, where the design process is usually manual, requiring the time and effort of the planners. The polygons generated in the short-term planning should have the least possible variability among them, so that the restrictions of the processing plant are met. Drawing short-term polygons automatically, sequencing all ore blocks in each polygon, and connecting one polygon to another is the focus of this work. In order to do this, we made use of genetic and dynamic algorithms, developed in Python programming language and operationalized in SGeMS geostatistical software. Multiple iterations were generated for each of the individual advances of ore blocks, generating regions or polygons, and selecting the regions of lower variability of contents. The functions of distribution of contents of each advance were compared to the function of the distribution of global contents of the ore body, the results show that the polygons have distributions similar to the reference distribution, so it was possible to sequence the blocks operationally.
\end{abstract}

Keywords: Short-term planning; Block sequencing; Stochastic; Provability distribution function; Genetic Algorithm.

1 Eng. De Minas, Mestrando em Eng. De Minas, DEMIN/PPGE3M, UFRGS, Porto Alegre, Rio Grande do Sul, Brasil.

2 Eng. De Minas, Doutor em Eng. De Minas, Professor, DEMIN/PPGE3M, UFRGS, Porto Alegre, Rio Grande do Sul, Brasil.

3 Eng. De Minas, Doutor em Eng. De Minas, Professor, Dep. De Geologia/IGEO, UFRGS, Porto Alegre, Rio Grande do Sul, Brasil. 


\section{INTRODUÇÃO}

A sequência de extração dos blocos, obtido pelo planejamento de lavra de longo prazo realizado com estimativas baseadas apenas em furos de sondagem (procedimento de maior precisão e custo), pode ser insuficiente para resolução em períodos de tempo em escalas de tempo pequenas (1 mês de produção por exemplo). Já no planejamento de curto prazo em uma mineração a céu aberto, geralmente, ocorre uma reavaliação dos teores dos blocos, geralmente estimados por métodos geoestatísticos, utilizando dados obtidos de malhas adensadas na etapa de quasi-produção, proveniente de métodos de amostragem como canaletas, trincheiras ou pó de perfuratriz. Utilizando dessa informação de curto prazo, ao invés de utilizar um algoritmo de otimização de cava (VPL - Valor Presente Líquido), o comum é desenhar polígonos de minério exposto e liberado em volumes que possam satisfazer as necessidades da planta de beneficiamento. Ainda, leva-se em conta os fatores restritivos associados à etapa de lavra, como os operacionais, tecnológicos, ambientais e ainda a incerteza geológica dos corpos minerais.

Como em muitas vezes não há o uso de algoritmos de otimização no planejamento curto prazo, sendo este feito de forma manual, o número de iterações para obter a meta de qualidade do minério (teor do minério e contaminantes dentro de limites estabelecidos) é limitado.

Ao realizar planejamento de curto prazo de forma manual, o planejador de lavra de curto prazo pode ser ver obrigado a não suprir de forma satisfatória a demanda de minério com as características tecnológicas demandadas pela planta de beneficiamento. Um outro ponto a ser observado, é que mesmo satisfazendo o teor médio de determinado período (semana, mês), é possível que ocorra grandes flutuações dos teores, levando a uma queda de rendimento da planta de beneficiamento. Normalmente, pode provocar problemas com a planta de beneficiamento, devido à alta variabilidade entre os polígonos, o que causa maiores custos nos processos de tratamento do minério.

Desta forma, uma alternativa é utilizar um algoritmo que use como referência a função de distribuição de probabilidades dos teores globais do minério (Ribeiro, 2007[1]), para as funções das distribuições de cada polígono. Note que, os polígonos são subconjuntos do minério global.

Neste contexto, este trabalho aborda uma estratégia para entregar uma solução simples, operacional e rápida ao problema de variabilidade do teor, desenvolvendo um algoritmo que estacionariza a variabilidade e sequencia os blocos dos polígonos. A proposta pode ser usada tanto em modelos de blocos krigados ou simulados, para o curto prazo.

\subsection{OBJETIVOS}

Este trabalho tem como objetivo aplicar um algoritmo de otimização de polígono no planejamento de curto prazo, bem como sequenciar a ordem de extração do material na frente de lavra. Os polígonos deverão apresentar a menor variabilidade possível entre eles. A otimização baseia-se na estacionarização dos teores, utilizando a pdf (probablility distribuction function), como referência. Ou seja, procura-se a sequência que garante flutuações mínimas nos histogramas de cada período analisado.

O Algoritmo é baseado em técnicas de otimização com algoritmos genéticos (Whitley, 1994[2]), e aplicados ao modelo de curto prazo conhecido popularmente 
como "modelo casca". Inicialmente, o trabalho foi desenvolvido para otimizar a sequência de lavra dos polígonos baseado nos teores estimados por krigagem (Matheron, 1963[3]).

\section{MATERIAIS E MÉTODOS}

Inicialmente, tem-se o modelo de curto prazo de determinado período (digamos mensal), ou modelo casca. Como deve-se garantir um controle de qualidade do material que alimenta a usina de beneficiamento (nesse caso, teor médio constante), que será lavrado, separa-se os blocos em minérios e estéril. A segunda etapa, é realizado uma definição do ponto inicial de lavra (ou múltiplos pontos), de onde partirá uma sequência de lavra dos blocos de forma aleatória (respeitando parâmetros de lavra e tendo um número pré-determinado de blocos). Um conjunto de cenários com o mesmo ponto de início, e mesmo número de elementos são gerados, seguindo caminhos aleatórios diferentes. Os cenários são submetidos a parâmetros de escolha (equações fitness), só escolhendo o cenário que melhor atenda as definições de qualidade do material proveniente da mina, e descartando os cenários restantes. Assim obtemos a população de blocos do primeiro polígono. A população de blocos de minérios é atualizada retirando o primeiro saque. Assim é inicializado um novo grupo de cenários para a definição do segundo polígono, desde o último bloco lavrado do primeiro polígono, escolhendo o saque mais apto e descartando o resto de cenários. Todo o processo é repetido até selecionar todos os blocos minérios adjacentes. Obtendo saques com as melhores representados pelos melhores cenários.

Para garantir que os parâmetros desejados de lavra estão sendo satisfeitos, é realizada uma comparação quantil/quantil usando a distribuição dos teores da população de blocos minérios (modelo global) e as distribuições de teores dos cenários gerados (iterações), para cada polígono (ou conjunto de polígonos). Medese os resíduos quadráticos e escolhe-se a solução que produzir o mínimo resíduo quadrático médio. Dessa forma, podemos garantir que a distribuição dos teores da sequência de lavra do curto prazo está os mais próximo possível da distribuição dos teores globais do depósito, garantindo a estacionariedade da qualidade do material a alimentar a planta de beneficiamento.

A erosão (ou sequência de lavra) é feita desde o(s) bloco(s) inicia(is) ou sementes, em forma de cruz, e erodindo randomicamente os blocos, possibilitando que os cenários obtidos sejam diferentes, ainda de só escolher blocos adjacentes. Para a implementação computacional e faz-se uso do algoritmo Union Find (Tarjan, 1975 [4]), para a erosão e sequenciamento dos blocos, complementado com a lógica de algoritmos genéticos.

A (Figura 1) ilustra o processo de erosão considerando os caminhos randômicos, onde as sementes estão simbolizadas por $k$ valores. P1, P2, ..., PT-1, PT representam os avanços ou saques. $S_{1}, S_{2}, S_{3}, \ldots, S_{n}$ representam os cenários gerados para cada avanço. Para o saque $\mathrm{P} 1$, foi selecionado o cenário $\mathrm{S} 3$ como 0 cenário ótimo. Continuando o processo em P2, e assim por diante. 


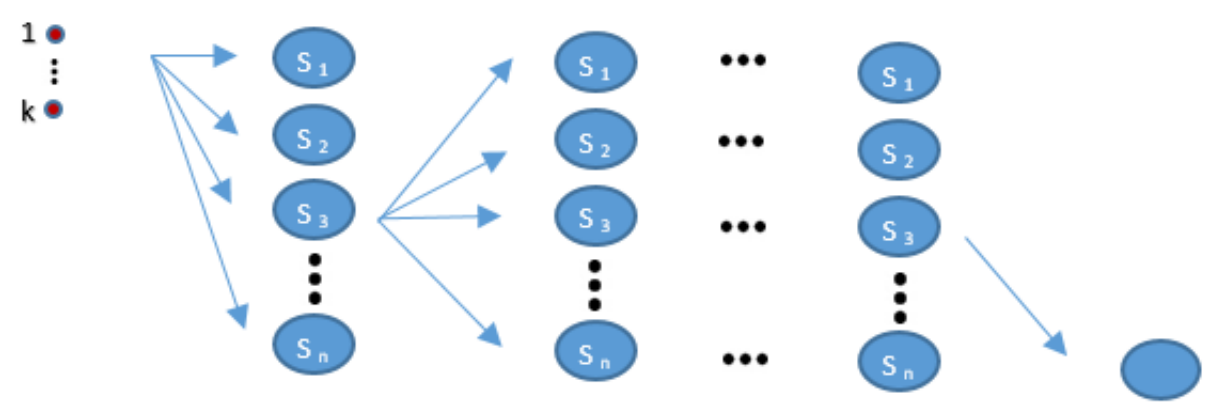

Figura 1. Esquema de sequenciamento dos polígonos do algoritmo

Como resultado final, temos o caminho randômico, que garanta a qualidade desejada (ou próximo disso), na alimentação da planta de beneficiamento, garantindo o ponto ótimo de funcionamento da mesma.

\section{RESULTADOS E DISCUSSÃO}

\subsection{ESTUDO DO CASO}

Para ilustrar a metodologia e o algoritmo, foi utilizado o software geoestatístico SGeMS (Remy, N., Boucher, A., \& Wu, J, 2006[5]). Para um estudo de caso em 2D, com a variável $\mathrm{Cu}$ (sintético) do Walker Lake, criou-se um modelo de blocos estimados, com dimensões de $5 \times 5 \times 1 \mathrm{~m}$ em x, y, z respectivamente. Totaliza 2900 blocos estimados (Figura 2) e teor de corte de $1.5 \%$.
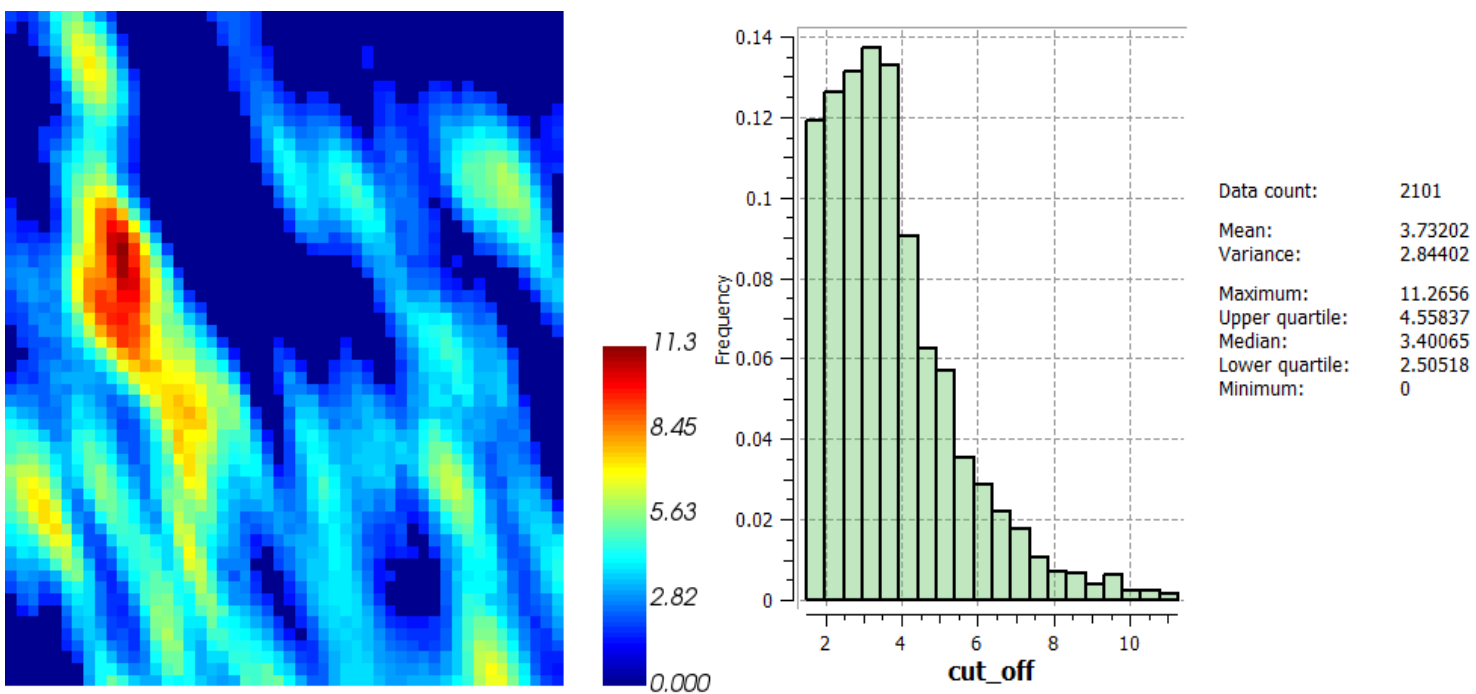

Figura 2. Mapa dos teores de minério acima ou igual ao 1.5 de teor, os outros abaixo de 1.5 são considerados zero (estéril). A direita, o histograma dos teores dos blocos de minério.

O histograma da (Figura 2), será considerado o histograma global a ser usado como a referência para a distribuição dos polígonos que serão desenhados. A média dos 
teores é de $3.73 \%$ e o desvio padrão é de $1.68 \%$. Dos 2900 blocos, $72.45 \%$ blocos são blocos de minério. Os blocos considerados estéreis representados em azul na escala de cores possuem valor zero.

Se assumimos a densidade de minério de $2 \mathrm{t} / \mathrm{m}^{3}$ e cada bloco tem um volumem de $25 \mathrm{~m}^{3}$, o requerimento da planta será de $20000 \mathrm{t} /$ dia, que representam os 400 blocos.

Para a utilização do algoritmo, alguns parâmetros que emulem a lavra dos polígonos são necessários, sendo eles apresentados na (Tabela 1).

Tabela 1. Dados de entrada

\begin{tabular}{lc}
\multicolumn{1}{c}{ Dados de entrada } & Valores \\
\hline Teor de corte (\%) & 1,5 \\
\hline Número de blocos de minério para cada avanço & 400 \\
\hline Número de iterações realizadas & 50 \\
\hline Número de avanços & 6 \\
\hline Número de sementes & 2 \\
\hline Local das sementes $(x, y, z)$ em metros & $(55,250,1) ;(210,237,1)$ \\
\hline
\end{tabular}

A (Figura 3) apresenta o primeiro avanço selecionado a partir de 50 cenários gerados, já que sua distribuição é a que mais se assemelha à distribuição de referência (global), e inicializado nas coordenadas descritas na (Tabela 1).
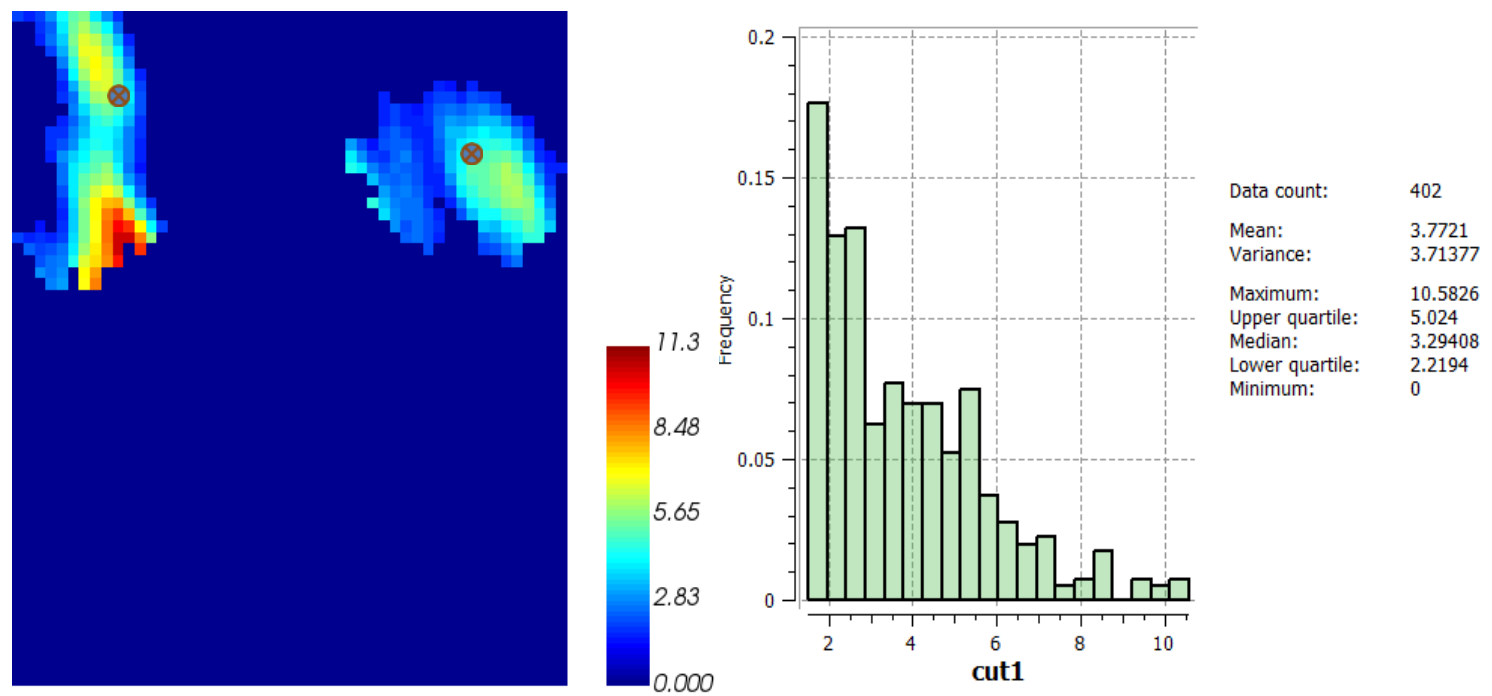

Figura 3. Mapa do primeiro avanço de minério com as respectivas sementes e a direita o histograma dos teores dos blocos minérios. 

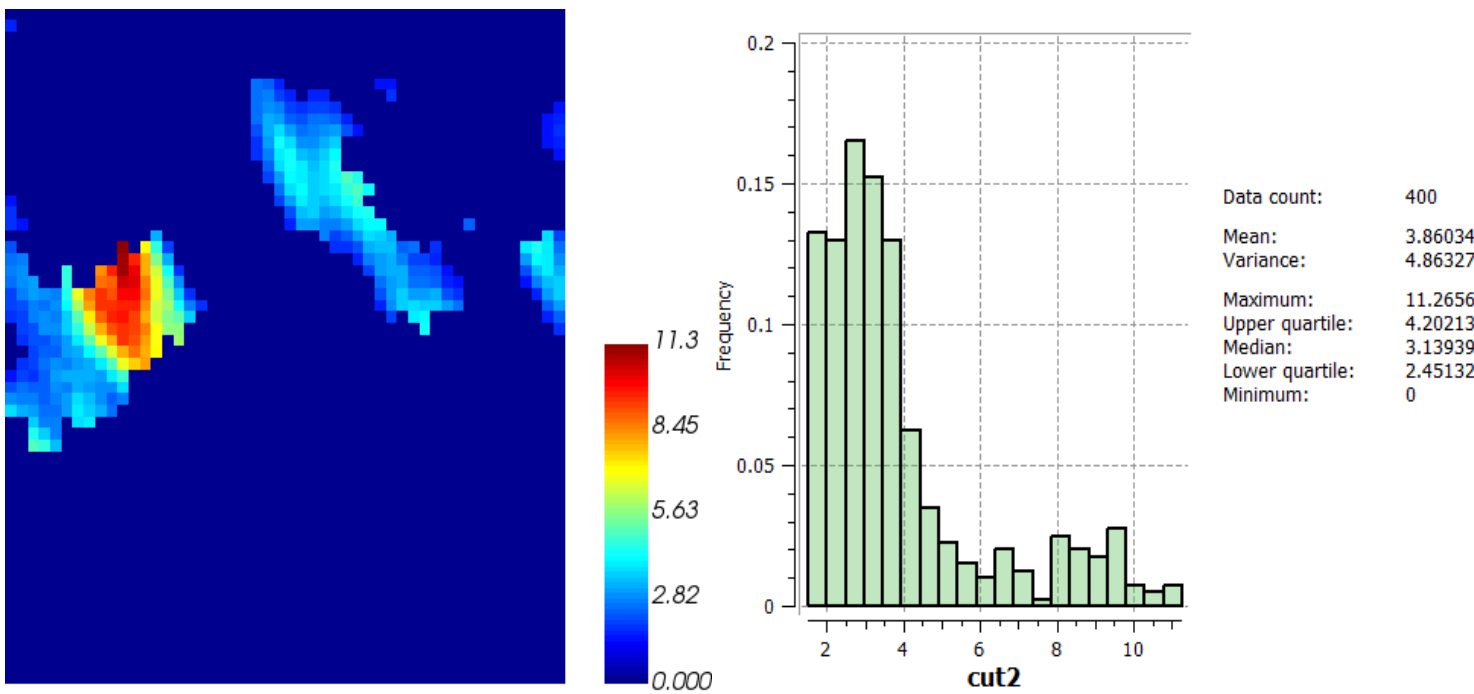

Figura 4. Mapa do segundo avanço de minério e a direita o histograma dos teores dos blocos minérios

O segundo polígono é iniciado no último bloco do primeiro avanço, sequenciado pelo algoritmo, e são feitas outras 50 realizações dos próximos 400 blocos. Seleciona-se o melhor cenário em termos de semelhança ao histograma global dos teores e assim sucessivamente até que todos os blocos de minério tenham sido lavrados.
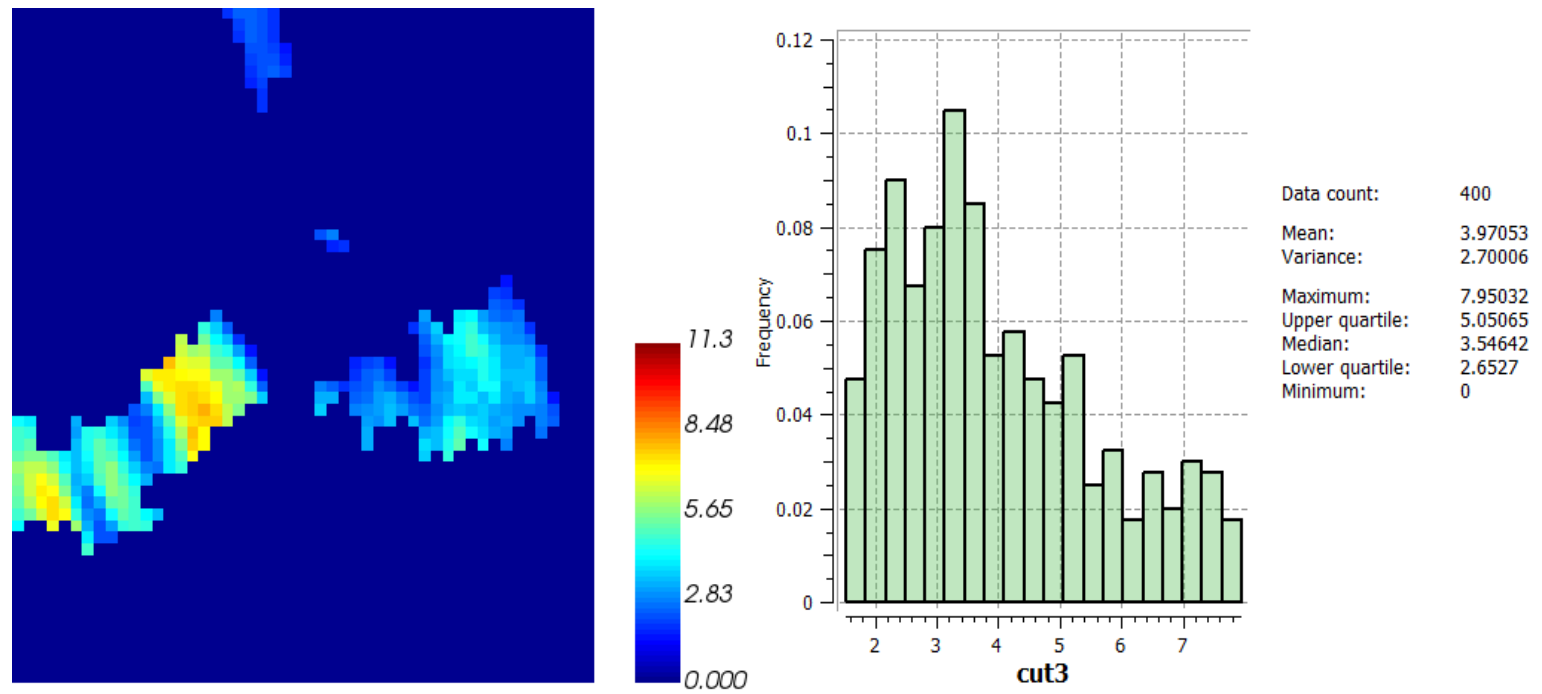

Figura 5. Mapa do terceiro avanço de minério. A direita, o histograma dos teores dos blocos minérios

O terceiro polígono é iniciado no último bloco do segundo avanço, sequenciado pelo algoritmo, e são feitas outras 50 realizações, dos próximos 400 blocos, selecionando a melhor cenário. 

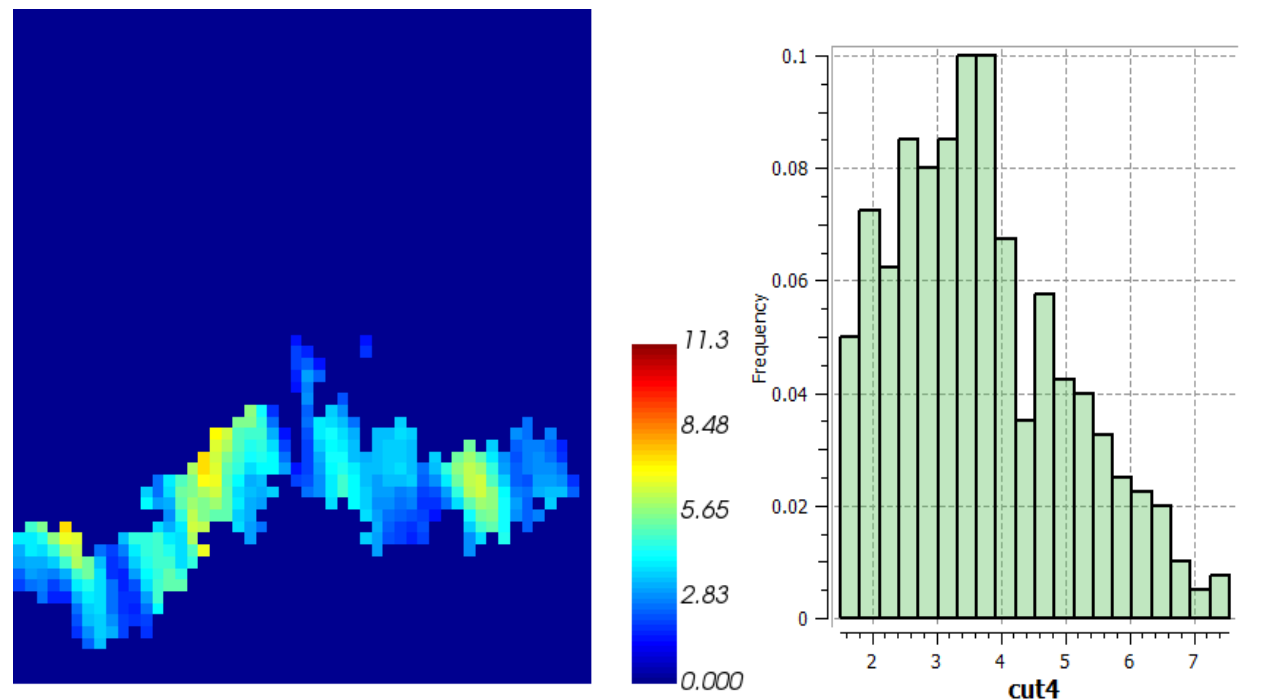

Data count: $\quad 400$

Maximum: $\quad 7.55523$

Upper quartile: $\quad 4.6018$

Median: 3.52883

Lower quartile: $\quad 2.63249$

Minimum:

Figura 6. Mapa do quarto avanço de minério e o histograma dos teores dos blocos de minério selecionados.
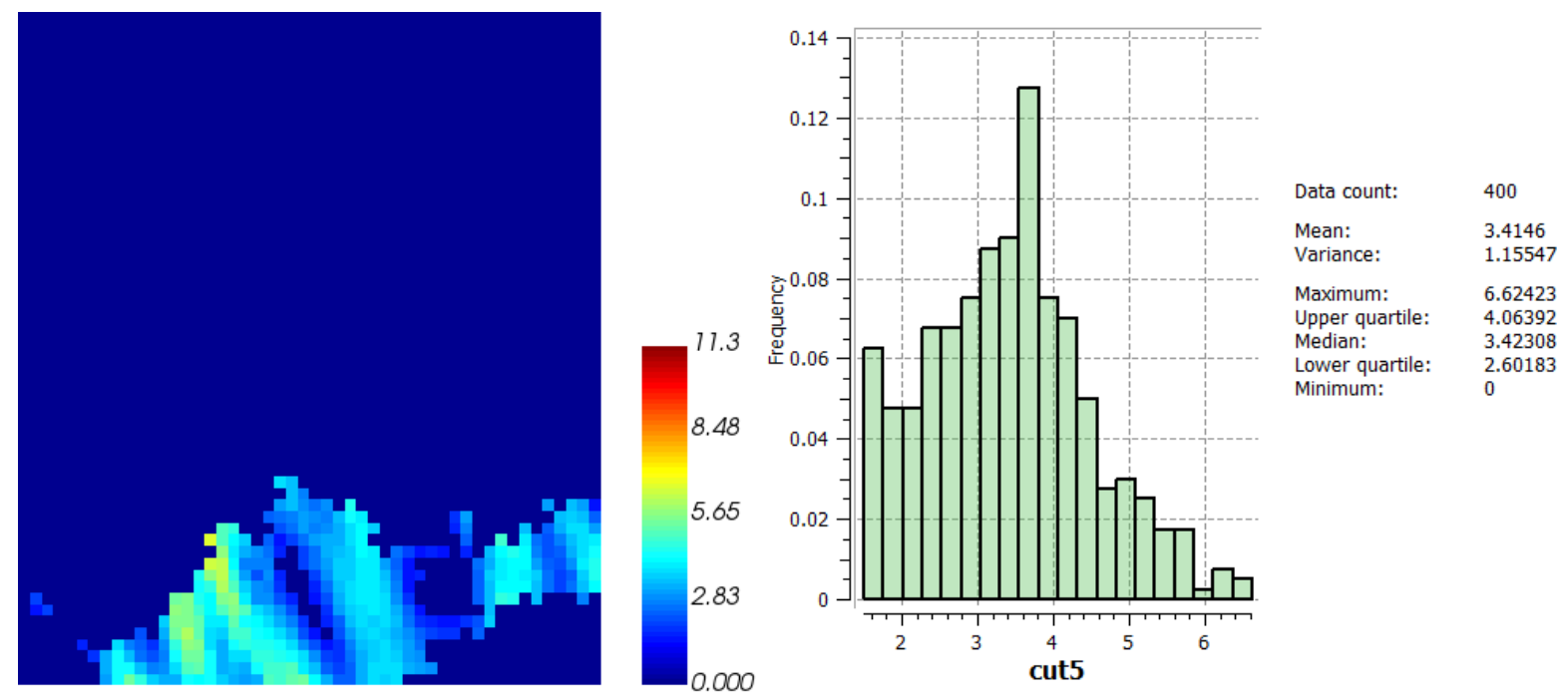

Figura 7. Mapa do quinto avanço de minério e o histograma dos teores dos blocos de minério selecionados.

O quarto polígono é iniciado no último bloco do terceiro avanço sequenciado pelo algoritmo, e são feitas outras 50 realizações dos próximos 400 blocos. Segue-se retendo o melhor cenário em semelhança a distribuição dos teores globais até serem lavrados todos os blocos. 
Tabela 2. Resumos das estatísticas

\begin{tabular}{ccccccc}
\hline Global & Media & Variância & $\begin{array}{c}\text { Desvio } \\
\text { Padrão }\end{array}$ & $\begin{array}{c}\text { Valor } \\
\text { Mínimo }\end{array}$ & $\begin{array}{c}\text { Valor } \\
\text { Máximo }\end{array}$ & $\begin{array}{c}\text { Número } \\
\text { blocos }\end{array}$ \\
\hline Avanço 1 & 3.73 & 2.84 & 1.69 & 1.5 & 11.27 & 2101 \\
\hline Avanço 2 & 3.86 & 3.71 & 1.93 & 1.5 & 10.58 & 402 \\
\hline Avanço 3 & 3.97 & 2.86 & 2.20 & 1.5 & 11.26 & 400 \\
\hline Avanço 4 & 3.69 & 1.78 & 1.64 & 1.5 & 7.95 & 400 \\
\hline Avanço 5 & 3.41 & 1.15 & 1.07 & 1.5 & 7.56 & 400 \\
\hline Avanço 6 & 3.61 & 2.14 & 1.42 & 1.5 & 6.62 & 400 \\
\hline
\end{tabular}

Note que o número de blocos do avanço 1 tem mais dois blocos em comparação aos demais avanços. Esses dois blocos excedentes representam os locais onde foram locadas as sementes. No avanço 6 (último), tem-se somente 96 blocos, que são os blocos restantes.

$\mathrm{Na}$ (Figura 8), observa-se que os blocos foram sequenciados a partir das coordenadas das sementes até lavrar-se todos os blocos de minério. Note, que os pontos sementes representam o início de lavra das escavadeiras e pode-se observar a sequência de avanço das escavadeiras.

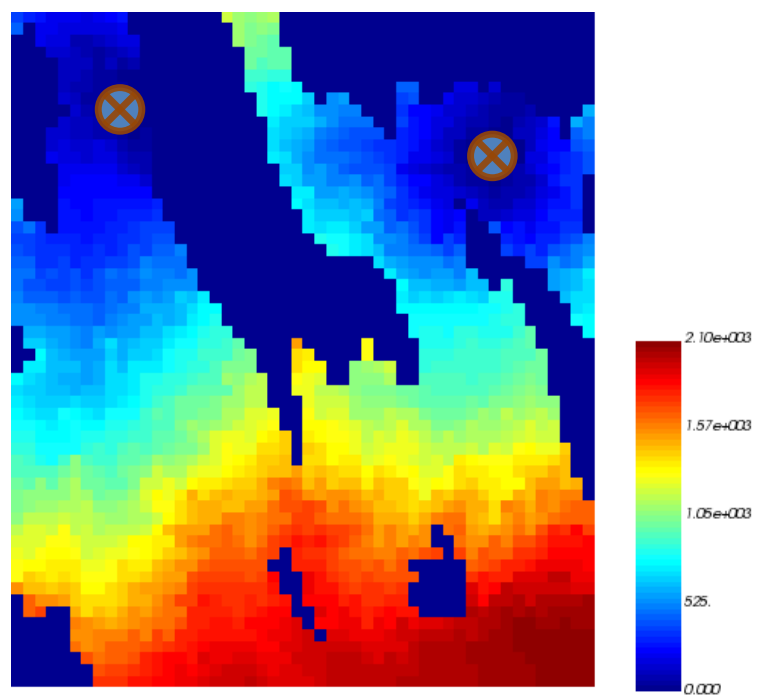

Figura 8. Mapa do sequenciamento dos blocos minérios, as cruzes indicam o local das sementes. A escala das cores apresenta os primeiros blocos nas faixas azuis e os últimos blocos nas faixas vermelhas. No meio, passa através de azul-claro, verde, amarelo, laranja.

\subsection{VALIDAÇÃO DOS RESULTADOS}

Para validar os resultados, faz-se uso do q-q plot onde os quantis de uma distribuição são plotados contra os quantis de outra distribuição (Isaaks, E. H., \& Srivastava, R. M, 1998 [6]). Uma distribuição será idêntica a outra quando os pontos que representam os quantis formem uma linha de 45 graus. Os gráficos da figura 10 mostram que as distribuições de teores dos avanços apresentam uma semelhança com a distribuição global de teores. 

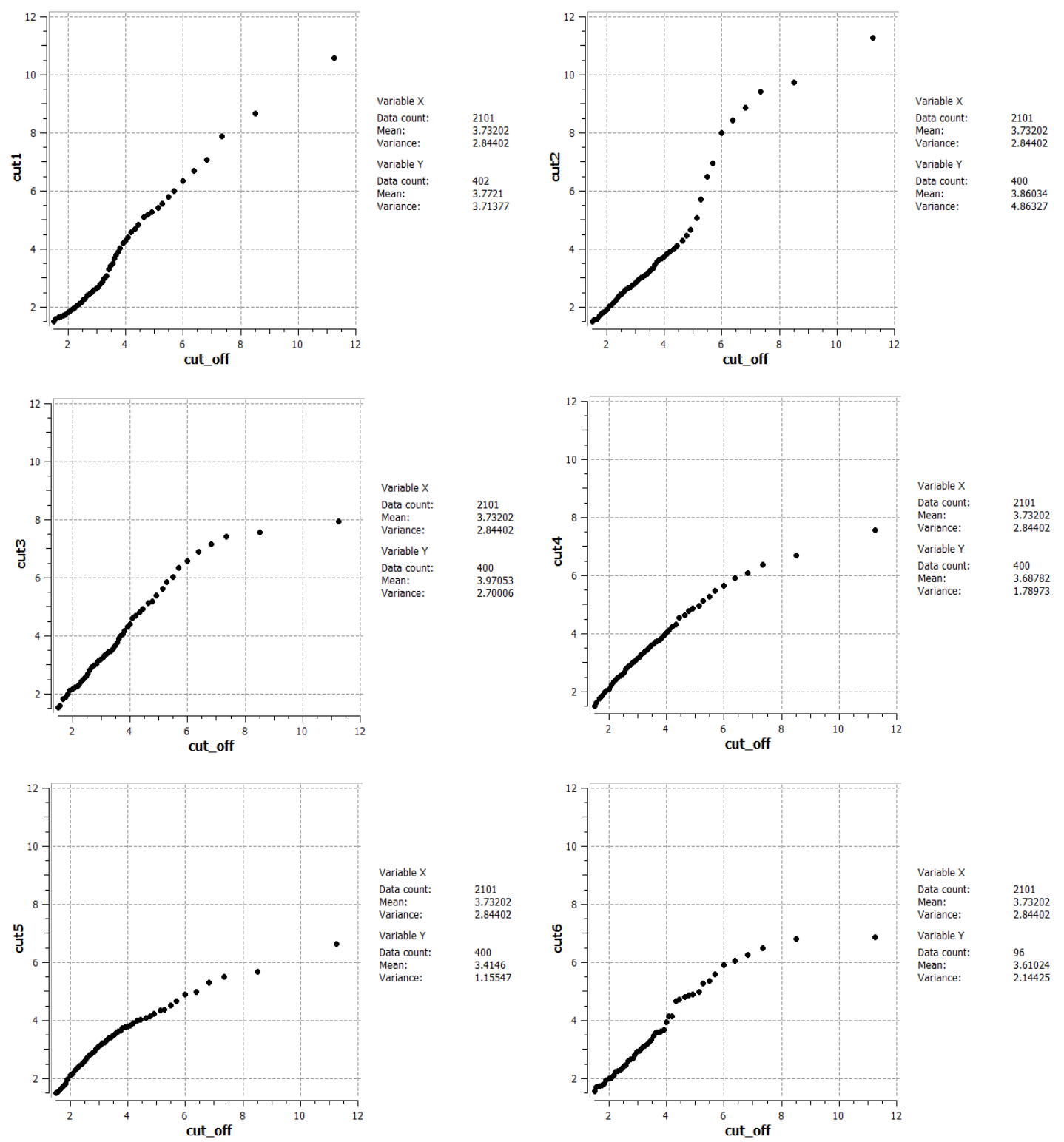

Figura 10. Gráficos q-q plot da distribuição global cut_off no eixo X, VS as distribuições dos avanços ou cut, no eixo $Y$

Observe-se que todos os avanços (cut), reproduzem de forma satisfatória os teores globais nas faixas de teores mais baixos e médios. Houve maior dificuldade de achar, em cada avanço, os teores altos na proporção que aparecem na distribuição global.

\section{CONCLUSÃO}

A utilização de algoritmos genéticos de otimização de planejamento de lavra no curto prazo mostra que é possível automatizar rotinas de trabalhos até então realizadas de forma manual, mas com maior eficiência e garantia da qualidade do produto a ser entregue na planta de beneficiamento. Além disso, permite uma maior combinação de parâmetros técnicos a ser analisados, como o número e a localização de escavadeiras a serem utilizadas, conforme à atualização da base de dados e a disponibilidade mecânica, respetivamente. 
A utilização de um parâmetro de controle como o histograma (comparativo entre o global e os locais) é uma técnica que dificilmente poderia ser aplicado sem um método de otimização numérica. Isso garante ao processo de planejamento uma metodologia menos laboriosa e mais eficiente.

O algoritmo pode ser usado em diferentes frentes de lavra simultaneamente, podendo ser utilizado para estimar uma homogeneização de materiais in situ, permitindo o aproveitamento de materiais que por si só não seriam caracterizados minérios.

Apesar do trabalho inicialmente ter sido feito com base em estimativas (no caso, krigagem), acredita-se que o uso de simulações estocásticas dos teores para analisar a incerteza associada a lavra de curto prazo seja benéfico, sendo essa a próxima etapa do estudo.

\section{Agradecimentos}

À CAPES pela bolsa de pós-graduação, de março 2016/2017, que permitiu a realização deste estudo.

\section{REFERÊNCIAS}

1 RIBEIRO, D. T. PDFseq Mine Scheduling Simulation Based on Block Probability Distribution Function An Iron Ore Application. 33 $3^{\text {th }}$ International Symposium on Computer Applications in the Mineral Industries. 2007; 33(1):369-374.

2 Darrell Whitley. A genetic algorithm tutorial. Statistics and Computing. 1994;4: 65-85.

3 Matheron, Georges. Principles of geostatistics. Economic geology.1963; 58(8): 12461266.

4 Tarjan, R. E. Efficiency of a good but not linear set union algorithm. Journal of the ACM (JACM), 1975;22(2), 215-225.

5 Remy, N., Boucher, A., \& Wu, J. Applied geostatistics with SGeMS: A user's guide. Cambridge: Cambridge University Press; 2009.

6 Isaaks, E. H., \& Srivastava, R. M. Applied geostatistics. New York: Oxford University Press; 1998. 